\title{
Overexpression of the oil palm (Elaeis guineensis Jacq.) TAPETUM DEVELOPMENT1-like Eg707 in rice affects cell division and differentiation and reduces fertility
}

\begin{abstract}
The functional analysis of the TAPETUM DEVELOPMENT1-like analog Eg707 of oil palm was carried out in rice by over-expressing Eg707 under the control of a double cauliflower mosaic virus 35S promoter. Ectopic expression of Eg707 in rice induced dark green and matured compact brownish calli compared to pale wild type and negative control calli. Regenerated transgenic rice plants exhibited a reduction in organ size and plant height, rolled, erect leaves, less tillers, increased chlorophyll content, and reduced fertility with smaller green seeds. At the molecular level Eg707 overexpression caused an increase in the transcription of SAPK9, a SnRK2 protein kinase family member that is activated by ABA and hyperosmotic stress. Together, the results show that ectopic Eg707 expression influences cell division and differentiation, presumably via altered hormone homeostasis.
\end{abstract}

Keyword: Oil palm; Abscisic acid; Hormone signaling; Tissue culture 